\title{
INFLUENCIA DE LA ACTITUD HACIA LA MARCA Y SU CALIDAD PERCIBIDA EN LA ACTITUD HACIA SUS ANUNCIOS: ¿AFECTAN LA INTENCIÓN DE COMPRA?
}

\author{
Ugalde, Cecilia ${ }^{1}$
}

Universidad del Azuay

cugalde@uazuay.edu.ec

Material original autorizado para su primera publicación en la revista académica

REDMARKA. Revista Digital de Marketing Aplicado.

https://doi.org/10.17979/redma.2014.01.013.4872

Recibido: 24 Noviembre 2014

Aceptado 9 Diciembre 2014

\section{Resumen}

El presente estudio pretende explorar la influencia y relación que la actitud hacia una marca así como la calidad percibida de un producto o servicio tienen hacia sus anuncios publicitarios, y cómo cada uno de ellos influye, si lo hace, en la intención de compra de los consumidores. El estudio se centra en el área de servicios, partiendo de la recordación de un anuncio publicitario en el segmento restaurantes.

Esta investigación explora entre otras una relación sobre la que no se encontraron estudios anteriores, la relación entre la calidad percibida de un producto o servicio y la actitud hacia sus anuncios publicitarios. Para esto se

\footnotetext{
${ }^{1}$ Candidata doctoral del Doctorado de Marketing de la Universidad de Valencia (España), Máster en Publicidad y Relaciones Públicas en Marquette University (Milwaukee, Wisconsin - Estados Unidos). Áreas de investigación centradas en temas relacionados con las comunicaciones integradas al marketing, en particular apego y personalidad de la marca, también en temas de comunicación orientados a competencias mediáticas. Docente investigadora de la Universidad del Azuay en Cuenca, Ecuador.
} 
realizaron 250 encuestas al azar en la ciudad de Cuenca, para cuyo análisis se utilizó SPSS y EQS.

Con base a este estudio se puede afirmar que la calidad percibida de una marca influye en la actitud hacia sus anuncios; mientras que la actitud hacia dicha marca no influye en la actitud hacia sus anuncios, pero sí lo hace en la intención de compra, lo que se presta para que a través de anuncios publicitarios se pueda mejorar la actitud hacia la marca si esta se ha visto dañada.

El hecho de que la actitud que se tiene hacia un anuncio publicitario no desencadene en intención de compra, refuerza la necesidad de explorar factores que mejoren la efectividad de los anuncios publicitarios.

Palabras clave: Publicidad, calidad percibida, intención de compra.

\section{Abstract}

This study aims to explore the influence and relationship that brand attitude and perceived quality of a product or service have over their advertisements, and how each influences, if so, in the consumer's purchase intent. The study focuses on the services area, based on the recall of an advertisement in the food service segment.

This research explores among others, a relationship about which we found no previous studies, that is the relationship between the perceived quality of a product or service and the attitude towards its advertisements. For this study 250 random surveys were conducted in the city of Cuenca. SPSS and EQS were used for the data analysis.

Based on this study it can be stated that the perceived quality of a brand influences the attitude towards its ads; while the attitude toward said brand does

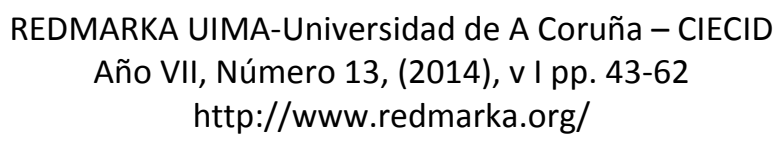


not influence the attitude towards its ads, but does influence in its purchase intent, which lends itself to that through advertising the attitude towards the brand can improve if it has been damaged.

The fact that the attitude you have towards an advertisement does not trigger on purchase intent, reinforces the need to explore factors that improve the effectiveness of advertisements.

Keywords: Advertising, perceived quality, purchase intent.

\title{
Resumo
}

Este estudo tem como objetivo explorar a influência e relacionamento que a atitude em relação a marca e a qualidade percebida do produto ou serviço tem que seus anúncios, e como cada influências, em caso afirmativo, na intenção de compra dos consumidores. O estudo centra-se na área de serviços, com base na lembrança de um anúncio publicitário no segmento dos restaurantes.

Esta investigação explora relação entre si sobre o qual não foram encontrados estudos anteriores, a relação entre a qualidade percebida de um produto ou serviço e atitude em relação a suas propagandas. Para este 250 pesquisas aleatórias foram realizadas na cidade de Cuenca, SPSS e EQS foi utilizado para análise de dados.

Com base neste estudo pode-se afirmar que a qualidade percebida de uma marca influencia a atitude para com os seus anúncios; enquanto que a atitude em relação à marca não influencia a atitude para com os seus anúncios, mas sim na intenção de compra, que se presta a que através da publicidade pode melhorar a atitude em relação à marca se foi danificado.

O fato de que a atitude que você tem para um anúncio publicitário não aciona na intenção de compra, reforça a necessidade de explorar os fatores que melhoram a eficácia dos anúncios.

\author{
REDMARKA UIMA-Universidad de A Coruña - CIECID \\ Año VII, Número 13, (2014), v I pp. 43-62 \\ http://www.redmarka.org/ \\ ISSN 1852-2300
}


Palavras-chave: Publicidade, qualidade percebida, intenção de compra.

\section{Introducción}

La actitud de los consumidores hacia los anuncios publicitarios puede verse afectada por una gran variedad de factores (Shen \& Chen, 2007, pág. 69), tema que ha generado interés en los investigadores desde los años setenta (Ej. Holbrook, 1978, pág. 545), y que ha sido ampliamente investigado como lo demuestran Brown y Stayman (1992) en el meta análisis que realizaron de los antecedentes y consecuencias de las actitudes hacia los anuncios publicitarios, así como en los numerosos estudios posteriores sobre el tema (Ej. Kim, Baek, \& Choi, 2012; Defever, Pandelaere, \& Roe, 2011), en los que se evidencia la importancia de la actitud hacia el anuncio publicitario y la gran cantidad de factores que en ella influyen.

Muchos de estos estudios revisados revelan que una actitud positiva hacia un anuncio publicitario puede trasladarse hacia la marca y hacia sus productos; lo que se desea explorar es si es que la actitud hacia un anuncio publicitario se ve afectado por la calidad percibida de la marca o por la actitud que previamente tenemos hacia ella.

\subsection{Calidad percibida}

La calidad percibida se considera como una dimensión del valor de marca, conjuntamente con lealtad de marca, apego a la marca y asociaciones de marca (Aaker, 1991, pág. 16; Yoo \& Donthu, 2001, págs. 5-7). La calidad percibida conjuntamente con el riesgo percibido, forman parte del concepto de valor percibido (Snoj, Korda, \& Mumel, 2004, pág. 163). De acuerdo con 
Zeithaml (1988, pág. 7), la calidad percibida de un producto, se relaciona con la actitud de un consumidor hacia la experiencia de marca en general, y no hacia características específicas del producto, el mismo autor define a la calidad percibida como "la evaluación total que el consumidor realiza de la excelencia o superioridad de un producto" (p.3). La calidad percibida al ser una variable de evaluación, se ha estudiado en modelos que explican intenciones de comportamiento (Gotlieb, Grewal, \& Brown, 1994, pág. 875).

\subsection{Actitud hacia la marca}

Desde los años sesenta encontramos estudios sobre la actitud hacia la marca (Myers, 1967), y cada vez más estudios conceptuales o empíricos dan mayor evidencia de los factores que afectan la actitud hacia la marca (Maclnnis, Rao, \& Weiss, 2002, pág. 395; Yoo \& Maclnnis, 2005). De manera especial encontramos estudios que analizan los efectos de los anuncios publicitarios en la actitud hacia la marca (Mitchell \& Olson, 1981; Gardner, 1985; Shen \& Chen, 2007); aunque varios autores identifican predictores cognitivos y afectivos de las actitudes hacia las marcas (Lutz, 1975, pág. 50), y dentro del campo afectivo, se han realizado varios estudios sobre el papel de las emociones y la evaluación de los anuncios publicitarios como predictores de la actitud hacia una marca (Brown, Homer, \& Inman, 1998, pág. 119).

\subsection{Intención de compra}

Estudios demuestran que la credibilidad hacia los anuncios publicitarios influye en la actitud que tenemos hacia las marcas, lo que a su vez influye en la intención de compra (MacKenzie \& Lutz, 1989, págs. 59-62; Goldsmith, Lafferty, \& Newell, 2000, pág. 46; Tucker, Rifon, Lee, \& Reece, 2012, pág. 10), otros autores afirman que el valor percibido es un determinante crítico de la intención de compra (Ej. Chang \& Wang, 2011, pág. 334; Beneke, Flynn, Greig, \& Mukaiwa, 2013, pág. 219), algunos se centran en la complejidad de los mensajes (Geissler, Zinkhan, \& Watson, 2006, pág. 70; Li, Daughety, \& Biocca, 2002, pág. 51) y otros incluyen a la intencion de compra en constructos 
complejos como el del valor de marca (Cobb-Walgren, Ruble, \& Donthu, 1995, pág. 29).

Spears y Singh (2004), definen la intención de compra como "el plan consciente de un individuo de hacer el esfuerzo para comprar una marca" (p.56).

\section{Cuestiones a investigar / hipótesis}

Por ser la calidad percibida una variable de evaluación que con frecuencia se incluye en modelos que explican intenciones de comportamiento, se decidió revisar si la calidad percibida afecta intenciones de comportamiento (Gotlieb, Grewal, \& Brown, 1994, pág. 875), en este caso orientadas hacia la intención de compra, relación que ya ha sido revisada en otros estudios (Ej. Beneke, Flynn, Greig, \& Mukaiwa, 2013), para lo que se formula la siguiente hipótesis:

H1: La calidad percibida de un producto o servicio influye de manera directamente proporcional en la intención de compra que tenemos de dicho producto o servicio.

Como ya se mencionó, se han realizado gran cantidad de estudios sobre los factores que influyen en la actitud hacia los anuncios publicitarios. De hecho, muchas de estas investigaciones se centran en una o más de estas relaciones (Brown \& Stayman, 1992, págs. 38-39), sin embargo, no se encontraron estudios que busquen o encuentren una relación entre la calidad percibida de un producto o servicio y la actitud que tenemos hacia sus anuncios publicitarios. Es probable que si tenemos una percepción positiva sobre la calidad de un producto o servicio, tengamos una actitud más favorable hacia sus anuncios publicitarios, es por eso que se plantea la siguiente hipótesis: 
H2: La calidad percibida de un producto o servicio influye de manera directamente proporcional en la actitud que tenemos hacia sus anuncios publicitarios.

Se hizo referencia a los muchos estudios que analizan la relación que la actitud hacia los anuncios publicitarios tiene en la actitud hacia la marca, sin embargo, no se encontró registro de estudios que analicen la relación a la inversa, es decir, la influencia que puede tener la actitud que tenemos hacia una marca en la actitud que nos genera su publicidad. Es posible que la actitud negativa hacia una marca nos predisponga frente a sus anuncios publicitarios, y que nuestras emociones influyan en cómo recibimos dicho mensaje, o a la inversa, que una actitud favorable hacia una marca influya en la receptividad y actitud con la que recibimos sus anuncios publicitarios, lo que afectaría en ambos casos la efectividad de dichos mensajes. Teniendo eso en mente, se plantea la siguiente hipótesis:

H3: La actitud hacia una marca influye de manera directamente proporcional en la actitud hacia sus anuncios publicitarios.

La actitud hacia la marca influye en la intención de compra (Ej. MacKenzie \& Lutz, 1989), se decidió medir si recordamos marcas hacia las que tenemos una actitud positiva y consecuentemente intención de compra, dentro de una categoría del área de servicios, para eso se planteó la siguiente hipótesis:

H4: La actitud hacia la marca recordada se relaciona positivamente con la intención de compra hacia la misma.

La intención de compra está estrechamente vinculada con los mensajes publicitarios (ej. MacKenzie \& Lutz, 1989) y la actitud que tenemos hacia ellos. En este estudio se explora si recordamos anuncios hacia los que tenemos una 
actitud positiva y consecuentemente intención de compra, dentro de una categoría del área de servicios, o por el contrario, recordamos aquello que nos impacta independientemente de que sea positivo o no, y no necesariamente vinculado con una actitud positiva que desencadene en intención de compra, con lo que se plantea la siguiente hipótesis:

H5: La actitud hacia la publicidad recordada se relaciona positivamente con la intención de compra hacia la misma.

En la figura 1 podemos observar el modelo causal propuesto con las relaciones planteadas.

Figura 1. Modelo Causal

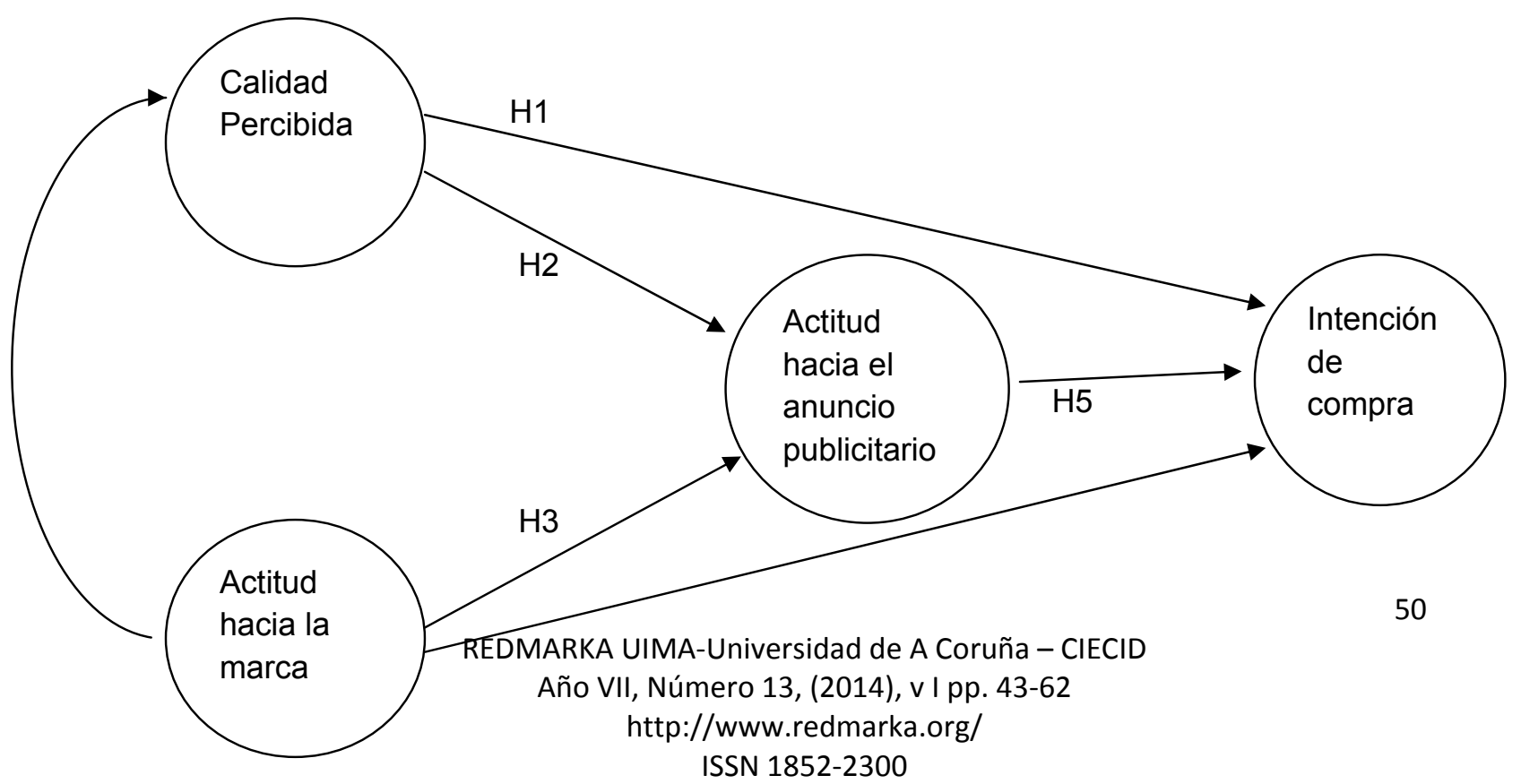




\section{Metodología}

Arbitrariamente se eligió la categoría restaurantes para realizar la investigación partiendo del principio de que todos nos alimentamos, y por lo tanto existe una alta posibilidad de que en determinado momento utilicemos este servicio. Se procedió a aplicar una encuesta a 250 personas interceptadas al azar en las calles céntricas de la ciudad de Cuenca. El cuestionario fue previamente probado con 15 personas, las encuestas se realizaron al medio día y al final de la tarde en días laborables.

El cuestionario se estructuró con preguntas cerradas con escalas Likert de 7 puntos. Únicamente la edad y el nombre del restaurante cuya publicidad recordaron en primer lugar fueron preguntas abiertas.

Las escalas de medida utilizadas se aplicaron con pequeñas modificaciones de sus originales, el detalle de las mismas con sus respectivos ítems y autores se encuentran en la tabla 1 , y el detalle del perfil de la muestra en la tabla 2.

Tabla 1. Escalas de medida utilizadas

\begin{tabular}{|c|c|c|c|}
\hline Autores, Año & Variables & & Cargas estandarizadas \\
\hline $\begin{array}{l}\text { Tucker, Rifon, Lee, \& Reece, } \\
2012 \quad \text { (adaptación } \\
\text { MacKenzie \& Lutz, 1989) }\end{array}$ & $\begin{array}{l}\text { Actitud hacia } \\
\text { anuncio }\end{array}$ & & $\begin{array}{l}\text { AA1 Mala/Buena } \\
\text { AA2 Desagradable/Agradable } \\
\text { AA3 Desfavorable/Favorable }\end{array}$ \\
\hline Yoo \& Donthu, 2001 & Calidad percibida & & $\begin{array}{l}\text { CP1 La calidad de este restaurante es muy } \\
\text { buena } \\
\text { CP2 Es muy alta la posibilidad de que en este } \\
\text { restaurante me vaya bien }\end{array}$ \\
\hline $\begin{array}{l}\text { Tucker, Rifon, Lee, \& } \\
2012 \quad \text { Reece, } \\
2012 \text { (adaptación }\end{array}$ & $\begin{array}{l}\text { Actitud } \\
\text { marca }\end{array}$ & la & AM1 Ir a este restaurante es una buena decisión \\
\hline
\end{tabular}


AM2 Creo que este es un restaurante satisfactorio

AM3 Tengo una actitud favorable hacia este restaurante

AM4 Me gusta este restaurante

AM5 Creo que el restaurante de la publicidad tiene muchas características positivas

IC1 Consideraría ir a este restaurante

IC2 Mi disposición de visitar este restaurante es alta

Tabla 2. Perfil de la muestra

\begin{tabular}{|c|c|c|}
\hline Género & Frecuencias & Porcentajes \\
\hline Femenino & 127 & 50.8 \\
\hline Masculino & 123 & 49.2 \\
\hline \multicolumn{3}{|l|}{ Edad } \\
\hline 13 a 18 años & 34 & 13.6 \\
\hline 19 a 29 años & 118 & 47.2 \\
\hline 30 a 45 años & 51 & 20.4 \\
\hline 46 a 60 años & 39 & 15.6 \\
\hline 61 a 85 años & 8 & 3.2 \\
\hline \multicolumn{3}{|l|}{ Actividad Principal } \\
\hline Estudiante & 113 & 45.2 \\
\hline Empleado público & 22 & 8.8 \\
\hline Empleado privado & 66 & 26.4 \\
\hline Negocio propio & 30 & 12 \\
\hline Ama de casa & 6 & 2.4 \\
\hline Jubilado & 4 & 1.6 \\
\hline Desempleado & 3 & 1.2 \\
\hline Otro & 6 & 2.4 \\
\hline \multicolumn{3}{|c|}{$\begin{array}{l}\text { Nivel más alto de educación } \\
\text { finalizado }\end{array}$} \\
\hline Escuela & 17 & 6.8 \\
\hline Colegio & 126 & 50.4 \\
\hline Universidad & 84 & 33.6 \\
\hline Maestría & 19 & 7.6 \\
\hline Doctorado & 4 & 1.6 \\
\hline \multicolumn{3}{|c|}{$\begin{array}{l}\text { Medio por el que recibió el mensaje } \\
\text { publicitario del restaurante que } \\
\text { recordó }\end{array}$} \\
\hline Radio & 30 & 12 \\
\hline TV & 27 & 10.8 \\
\hline Diario & 10 & 4 \\
\hline Revista & 8 & 3.2 \\
\hline Publicidad Exterior & 53 & 21.2 \\
\hline Hojas volantes & 19 & 7.6 \\
\hline
\end{tabular}

REDMARKA UIMA-Universidad de A Coruña - CIECID

Año VII, Número 13, (2014), v I pp. 43-62

http://www.redmarka.org/

ISSN 1852-2300 


\begin{tabular}{|c|c|c|}
\hline Estados de cuenta & 7 & 2.8 \\
\hline Redes Sociales & 36 & 14.4 \\
\hline Otros Internet & 6 & 2.4 \\
\hline Teléfono & 1 & 0.4 \\
\hline $\begin{array}{l}\text { Combinación de más de un } \\
\text { medio }\end{array}$ & 53 & 21.2 \\
\hline
\end{tabular}

\section{Resultados / Discusión}

Para validar la fiabilidad y validez de la encuesta se realizó un análisis factorial confirmatorio (AFC) una vez que se ingresaron los datos de las 250 encuestas en SPSS y se exportaron a EQS, luego se estimó el modelo estructural (SEM) para el testeo de las hipótesis. Tanto para el AFC como el SEM se utilizó el software EQS para Windows (versión 6.2).

Antes de validar la fiabilidad y validez del instrumento de medida se analizó la bondad de ajuste para saber si el modelo encaja o no con la realidad. Para esto se analizó la matriz residual de covarianzas o cuadro de residuos, los que se encontraron agrupados hacia el centro en los numerales seis y siete, lo que implica que el ajuste es bueno. No se consideró al Chi cuadrado por ser un estadístico muy sensible al tamaño de la muestra y porque la hipótesis nula es muy restrictiva ya que los modelos no son más que una aproximación a la realidad.

El ajuste de acuerdo con el parámetro CFI (Comparative Fix Index) $=0.98$ es bueno ya que es superior a 0.95. En cuanto al RMSEA (Root Mean Square of Error Aproximation) $=0.06$, no es perfecto, pero se encuentra dentro de la zona de tolerancia. En la tabla 3 se observa la validación de las medidas del modelo, donde queda en evidencia que todas las cargas de los factores son superiores a 0.7 y todas las $t$ tienen cargas factoriales significativas, por lo que 
es un estadístico muy sensible al tamaño de la muestra, en todo caso, los demás parámetros (CFI y RMSEA) están dentro de los índices deseados, lo que corrobora la que la bondad de ajuste es buena.

En cuanto al testeo de las hipótesis, los datos arrojan que no existe una carga factorial significativa en la primera hipótesis, a pesar de que la relación entre calidad percibida e intención de compra ya ha sido probada en otros estudios (Beneke, Flynn, Greig, \& Mukaiwa, 2013) como se mencionó al plantear la hipótesis. Esta situación puede ocurrir en función con la categoría de servicio estudiada, o debido a que el estudio gira alrededor de la actitud hacia anuncios publicitarios, en todo caso se abren opciones de investigación en este campo.

Al examinar la $\mathrm{H} 2$, se comprueba que sí existe una relación entre la calidad percibida y la actitud hacia el anuncio, relación de la que no se encontraron datos en la bibliografía revisada. Esto indicaría como ya se especuló, que si tenemos una percepción positiva sobre la calidad de un producto o servicio, tengamos una actitud más favorable hacia sus anuncios publicitarios, por lo que la $\mathrm{H} 2$ es aceptada. No ocurre lo mismo con la $\mathrm{H} 3$, ya que los datos demuestran que la actitud hacia la marca no influye en la actitud hacia el anuncio. Esto resulta interesante ya que implicaría que marcas sobre las que se tiene una actitud negativa pueden intentar cambiar la misma a través de sus anuncios publicitarios ya que la actitud hacia ellos no se ve afectada por la de la marca y encontramos muchos estudios que demuestran que más bien es la actitud hacia el anuncio la que influye en la actitud hacia la marca como ya se mencionó con anterioridad en este trabajo.

La H4 también se corrobora por lo que se ratifica que la actitud hacia la marca recordada se relaciona con la intención de compra hacia la misma, lo cual tiene sentido y explica de alguna manera el hecho de que se recuerden de manera selectiva ciertas publicidades sobre otras.

\footnotetext{
REDMARKA UIMA-Universidad de A Coruña-CIECID

Año VII, Número 13, (2014), v I pp. 43-62

http://www.redmarka.org/
}

ISSN 1852-2300 
Finalmente, la H5 se rechaza, en vista de que no se obtuvo una carga factorial significativa. Esto implica que la actitud que se tiene hacia un anuncio publicitario no desencadena en intención de compra, situación que se debe considerar cuidadosamente por las claras repercusiones que puede tener en inversión publicitaria y en el cumplimiento de sus objetivos.

Tabla 4. Testeo de Hipótesis

\begin{tabular}{|c|c|c|c|}
\hline \multicolumn{4}{|c|}{ Testeo de Hipótesis } \\
\hline \multicolumn{2}{|c|}{ Hipótesis } & $\beta$ & Valor $\mathbf{t}$ \\
\hline H1 & Calidad percibida $\longrightarrow$ Intención de compra & 0.338 & 1.763 \\
\hline $\mathrm{H} 2$ & Calidad percibida $\longrightarrow$ Actitud hacia el anuncio & $0.880^{* *}$ & 3.323 \\
\hline $\mathrm{H} 3$ & Actitud hacia la marca $\longrightarrow$ Actitud hacia el anuncio & -0.279 & -1.110 \\
\hline H4 & Actitud hacia la marca $\longrightarrow$ Intención de compra & $0.490^{*}$ & 2.950 \\
\hline H5 & Actitud hacia el anuncio $\longrightarrow$ Intención de compra & 0.101 & 1.460 \\
\hline & $\begin{array}{l}48)=89.57 ;(p<.01) ; \text { RMSEA }(90 \% \mathrm{Cl})= \\
971 \\
.01 ;{ }^{*} p<.05\end{array}$ & $059(0.040,0$ & $\mathrm{CFI}=. \mathrm{S}$ \\
\hline
\end{tabular}

\section{Conclusiones / aplicaciones / limitaciones}

En conclusión se puede decir que de los resultados obtenidos posiblemente los más interesantes son la relación de la calidad percibida del producto o servicio con la actitud hacia el anuncio, así como la no influencia de la actitud hacia la marca en la actitud hacia el anuncio, lo que se presta como ya se dijo para que a través de anuncios publicitarios se pueda mejorar la actitud hacia la marca si esta se ha visto dañada.

Otro punto interesante es que la actitud que se tiene hacia un anuncio publicitario no desencadena en intención de compra, lo que refuerza la 
necesidad de explorar factores que mejoren la efectividad de los anuncios publicitarios.

La muestra podría mejorar si se intenta que refleje más las características de la sociedad en la que se aplica, lo cual es difícil a tratarse de una encuesta aplicada al azar en la calle.

La bondad del ajuste si bien es buena, también podría mejorar, para lo que se sugiere realizar una nueva investigación a una muestra más grande, aunque de manera exploratoria se obtuvieron resultados interesantes.

\section{Investigación futura}

La investigación deja muchas incógnitas, pero despiertan particular interés explorar la relación de otros factores como la percepción en la actitud hacia los anuncios; por ejemplo, ¿Qué sucede con la lealtad?, ¿Tenemos una mejor actitud hacia los anuncios publicitarios de aquella firmas hacia las que somos fieles?, o no afecta, somos fieles, pero no por eso dejamos de ser críticos. Otra relación interesante en el mismo sentido se da entre el apego a la marca y la actitud hacia sus anuncios, partiendo siempre del hecho de que la actitud hacia el anuncio nos coloca en una disposición más favorable hacia la compra de un producto o servicio.

Definitivamente otra línea a investigar apunta hacia cómo mejorar el impacto de los anuncios publicitarios en la intención de compra de los consumidores.

\section{Bibliografía}


Aaker, D. A. (1991). Managing Brand Equity. New York: The Free Press.

Beneke, J., Flynn, R., Greig, T., \& Mukaiwa, M. (2013). The influence of Perceived Product Quality, Relative Price and Risk on Customer Value and Willingness to Buy: A Study of Private Label Merchandise. Journal of Product \& Brand Management, 22(3), pp. 1-18. Recuperado el 9 de 5 de 2013, de http://dx.doi.org/10.1108/JPBM-02-2013-0262

Brown, S. P., \& Stayman, D. M. (1992). Antecedents and Consequences of Attitude Toward the Ad: A Meta-Analysis. Journal of Consumer Research, 19(1), pp. 34-51. Recuperado el 09 de 05 de 2013, de http://www.jstor.org/stable/2489186

Brown, S. P., Homer, P. M., \& Inman, J. J. (1998). A meta-analysis of relationships between ad-evoked feelings and advertising responses. Journal of Marketing Research, 35(1), pp. 114-126. Recuperado el 23 de 9 de 2014, de http://www.jstor.org/stable/3151934

Chang, H. H., \& Wang, H.-W. (2011). The moderating effect of customer perceived value on online shopping behavior. Online Information Review, 35(3), pp. 333-359. Recuperado el 9 de 5 de 2013, de http://www.emeraldinsight.com/doi/full/10.1108/14684521111151414

Cobb-Walgren, C. J., Ruble, C. A., \& Donthu, N. (1995). Brand Equity, Brand Preference, and Purchase Intent. Journal of Advertising, 24(3), pp. 2540. Recuperado el 9 de 5 de 2013, de http://www.tandfonline.com/doi/pdf/10.1080/00913367.1995.10673481

Defever, C., Pandelaere, M., \& Roe, K. (2011). Inducing Value-congruent Behavior Through Advertising and the Moderating Role of Attitudes Toward Advertising. Journal of Advertising, 40(2), pp. 25-37. Recuperado el 09 de 05 de 2013, de 
http://www.tandfonline.com/doi/pdf/10.2753/JOA00913367400202\#.VHIT3ouG98E

Gardner, M. P. (1985). Does Attitude Toward the Ad Affect Brand Attitude Under a Brand Evaluation Set? Journal of Marketing Research, 22(2), pp. 192-198. Recuperado el 23 de 9 de 2014, de http://www.jstor.org/stable/3151364

Geissler, G. L., Zinkhan, G. M., \& Watson, R. T. (2006). The influence of home page complexity on consumer attention, attitudes, and purchase intent. Journal of Advertising, 35(2), pp. 69-80. Recuperado el 9 de 5 de 2013, de http://dx.doi.org/10.1080/00913367.2006.10639232

Goldsmith, R. E., Lafferty, B. A., \& Newell, S. J. (2000). The impact of Corporate Credibility and Celebrity Credibility on Consumer Reaction to Advertisements and Brands. Journal of Advertising, 29(3), pp. 43-54.

Recuperado el 23 de 9 de 2014, de http://dx.doi.org/10.1080/00913367.2000.10673616

Gotlieb, J. B., Grewal, D., \& Brown, S. W. (1994). Consumer Satisfaction and Perceived Quality: Complementary or Divergent Constructs? Journal of Applied Psychology, 79(6), pp. 875-885. Recuperado el 11 de 5 de 2013, de http://dx.doi.org/10.1037/0021-9010.79.6.875

Holbrook, M. B. (1978). Beyond Attitude Structure: Toward the Information Determinants of Attitude. Journal of Marketing Research, 15(4), pp. 545556. Recuperado el 09 de 05 de 2013, de http://www.jstor.org/stable/3150624

Kim, J., Baek, Y., \& Choi, H. Y. (2012). The Structural Effects of Metaphorelicided Cognitive and affective elaboration levels on attitude toward the ad. Journal of Advertising, 41(2), pp. 77-96. Recuperado el 09 de 05 de 
2013, de http://www.tandfonline.com/doi/pdf/10.2753/JOA00913367410206\#.VHISLouG98E

Li, H., Daughety, T., \& Biocca, F. (2002). Impact of 3-D Advertising on Product Knowledge, Brand Attitude, and Purchase Intetion: The Mediating Role of Presence. Journal of Advertising, 31(3), pp. 43-57. Recuperado el 23 de 9 de 2014, de http://dx.doi.org/10.1080/00913367.2002.10673675

Lutz, R. J. (1975). Changing brand attitudes through modification of cognitive structure. Journal of Consumer Research, 1(4), pp. 49-59. Recuperado el 25 de 9 de 2014, de http://www.jstor.org/stable/2488707

Maclnnis, D. J., Rao, A. G., \& Weiss, A. M. (2002). Assessing When Increased Media Weight of Real-World Advertisementes Helps Sales. Journal of Marketing Research, 39(4), pp. 391-407. Recuperado el 23 de 9 de 2014, de doi: http://dx.doi.org/10.1509/jmkr.39.4.391.19118

MacKenzie, S. B., \& Lutz, R. J. (1989). An Empirical Examination of the Structural Antecedents of Attitude Toward the Ad in an Advertising Pretesting Context. Journal of Marketing, 53(2), pp. 48-65. Recuperado el 20 de 9 de 2014, de http://www.jstor.org/stable/1251413

Mitchell, A. A., \& Olson, J. C. (1981). Are product Attribute Beliefs the Only Mediator of Advertising Effects on Brand Attitudes? Journal of Marketing Research, 18, pp. 318-331. Recuperado el 23 de 9 de 2014, de http://muse.jhu.edu/login?auth=0\&type=summary\&url=/journals/asr/v001/ 1.1mitchell.html

Myers, J. G. (1967). Determinants of Private Brand Attitude. Journal of Marketing Research, 4(1), pp. 73-81. Recuperado el 13 de 5 de 2013, de http://www.jstor.org/stable/3150168

Shen, F., \& Chen, Q. (2007). Contextual Priming and applicability: Implications for Ad Attitude and Brand Evaluations. Journal of Advertising, 36(1), pp. 
69-80. Recuperado el 09 de 05 de 2013, de http://dx.doi.org/10.2753/JOA0091-3367360105

Snoj, B., Korda, A. P., \& Mumel, D. (2004). The relationships among perceived quality, perceived risk and perceived product value. Journal of Product \& Brand Management, 13(3), pp. 156-167. Recuperado el 23 de 11 de 2014, de http://www.emeraldinsight.com/doi/pdfplus/10.1108/10610420410538050

Spears, N., \& Singh, S. (2004). Measuring attitude toward the brand and purchase intentions. Journal of Current Issues and Research Advertising, 26, pp. 53-66. Recuperado el 23 de 9 de 2014, de http://www.tandfonline.com/doi/pdf/10.1080/10641734.2004.10505164\#. VHKVpluG98E

Tucker, E. M., Rifon, N. J., Lee, E. M., \& Reece, B. B. (2012). Consumer Receptivity to Green Ads. Journal of Advertising, 41(4), pp. 9-23. Recuperado el 9 de 5 de 2013, de http://dx.doi.org/10.1080/00913367.2012.10672454

Yoo, B., \& Donthu, N. (2001). Developing and validating a multidimensional consumer-based brand equity scale. Journal of Business Research, 52 , pp. 1-14. Recuperado el 9 de 5 de 2013, de http://www.sciencedirect.com/science/article/pii/S0148296399000983

Yoo, C., \& MacInnis, D. (2005). The brand attitude formation process of emotional and informational ads. Journal of Business Research, 58, pp. 1397-1406. Recuperado el 23 de 9 de 2014, de http://www.sciencedirect.com/science/article/pii/S0148296305000640

Zeithaml, V. A. (1988). Consumer Perceptions of Price, Quality, and Value: A Means-End Model and Synthesis of Evidence. Journal of Marketing, 52(3), pp. 2-22. Recuperado el 9 de 5 de 2013, de 
Influencia de la actitud hacia la marca y su calidad percibida ...

Ugalde, Cecilia

http://www.jstor.org/discover/10.2307/1251446?uid=7519056\&uid=37379

$52 \&$ uid $=2 \&$ uid $=3 \&$ uid $=67$ \&uid $=62 \&$ uid $=40882 \&$ sid $=21104633036951$ 